Anita J. Reddy, MD, MBA

Department of Critical Care,

Respiratory Institute, Cleveland Clinic

Michael Cruise, MD, PhD

Pathology and Laboratory Medicine

Institute, Cleveland Clinic
Thomas G. Fraser, MD

Department of Infectious Disease,

Respiratory Institute, Cleveland Clinic
Purva Grover, MD

Emergency Services Institute,

Cleveland Clinic
Allison L. Weathers, MD

ITD Technology Services and Neurologic

Institute, Cleveland Clinic
Meredith A. Foxx, MBA, MSN, APRN

Nursing Institute, Cleveland Clinic
Christopher M. Babiuch, MD

Department of Family Medicine, Cleveland Clinic Community Care, Cleveland Clinic

\author{
Walter H. Henricks, MD \\ Pathology and Laboratory Medicine \\ Institute, Cleveland Clinic
}

\author{
Stephen W. Meldon, MD \\ Emergency Services Institute, \\ Cleveland Clinic
}

Ashleigh Muenzenmeyer, MSLA, SC(ASCP)

Pathology and Laboratory Medicine Institute, Cleveland Clinic
Shannon L. Pengel, RN, MSN

Nursing Institute, Cleveland Clinic
James F. Simon, MD

Medical Operations and Glickman Urological and Kidney Institute, Cleveland Clinic
Gary W. Procop, MD

Pathology and Laboratory Medicine Institute, Cleveland Clinic

\title{
Operationalizing COVID-19 testing: Who, what, when, where, why, and how
}

\section{Updated February 26, 2021}

\section{ABSTRACT}

The authors review the rationale behind and approaches to testing for COVID-19, the quality of currently available tests, the role of data analytics in strategizing testing, and using the electronic medical record and other programs designed to steward COVID-19 testing and follow-up of patients.

\section{WHY SHOULD WE TEST FOR COVID-19?}

Testing plays a key role in the diagnosis of COVID19. The critical importance of testing has never been more apparent than in the current pandemic. Testing for the hospitalized patient informs isolation practice, allowing a health system to optimize its use of personal protective equipment (PPE). Without testing, isolation practice would have to be syndromic, resulting in far more isolated patients and a significant increase in the use of PPE.

Ready access to testing also informs the strategy for maintaining a robust healthcare work force and mitigates the problem of presenteeism, ie, coming to work while ill. Outside the hospital, identifying infected patients is essential to control the spread of the virus in the community. Testing makes possible contact tracing by public health authorities who can then identify and isolate others who are ill and quar-

The statements and opinions expressed in COVID-19 Curbside Consults are based on experience and the available literature as of the date posted. While we try to regularly update this content, any offered recommendations cannot be substituted for the clinical judgment of clinicians caring for individual patients.

doi:10.3949/ccjm.87a.ccc048 antine those who are exposed. Testing also provides critical epidemiologic data that helps society understand current and future resource needs. ${ }^{1}$ Finally, the ultimate goal is for testing to identify patients who would benefit from targeted, effective treatment.

The role of testing has expanded from diagnosis to the screening of asymptomatic individuals. Screening patients before surgery and other procedures improves patient outcomes and makes it possible to use PPE more judiciously in these settings.

Often underappreciated is the effect of using even a highly sensitive and specific test in an asymptomatic population (ie, screening) in which the pretest probability of infection is low. In low-prevalence settings, the positive predictive value of even excellent tests is significantly lower, which ranslates into more false-positive test results. Confirmation or further investigation of positive test results in these settings may be warranted.

\section{WHAT ARE THE COVID-19 TESTS AVAILABLE, AND HOW GOOD ARE THEY?}

The US Centers for Disease Control and Prevention (CDC) and state public health laboratories were the only sites at which testing was available in the early stages of this pandemic in the United States. However, it soon became clear that these institutions could not handle the surge of testing that would come with the community spread of COVID-19. At this point, the US Food and Drug Administration (FDA) started to grant emergency use authorization to manufacturers and hospital laboratories. At the time of this writing, 
the FDA has granted emergency use authorization to 285 assays, most of which were for direct molecular detection, and a minority for serologic testing. ${ }^{2}$ More recently, the FDA has relaxed their requirement to review new emergency use authorizations for SARSCoV-2 assays. The laboratories of the Pathology and Laboratory Medicine Institute at Cleveland Clinic Health System perform multiple SARS-CoV-2 assays to meet clinical demand.

The CDC SARS-CoV-2 assay was the initial assay used at Cleveland Clinic, but it has been replaced by the TaqPath COVID-19 Combo Kit (ThermoFisher Scientific, Waltham, MA), which affords greater throughput (ie, more tests per shift) and better operational efficiency. This assay, like the CDC assay, requires a full nucleic acid extraction prior to reverse transcription and polymerase chain reaction (PCR)-based nucleic acid amplification. This assay is performed daily and on multiple shifts at Cleveland Clinic, with results from this test available within 24 hours from specimen receipt, and often sooner.

The second assay validated was the TIB MOLBIOL/Roche z 480 (TIB MOLBIOL, Adelphia, NJ and Roche Diagnostics, Indianapolis, IN) assay. This assay also has excellent performance characteristics (eg, a very low limit of detection). Based on studies performed at Cleveland Clinic, this assay has received FDA emergency use authorization. The results from this test are also available within 24 hours from specimen receipt. This assay will be used in conjunction with the Cleveland Clinic Home Collection kit.

Two automated solutions have also been added to the SARS-CoV-2 testing portfolio: the cobas SARSCoV-2 Test using the 6800/8800 systems (Roche Diagnostics, Indianapolis, IN) and the Aptima SARS-CoV-2 Assay (Panther System) (Hologic, Marlborough, MA). These assays have significantly expanded our testing capacity, and their automated features improved our operational efficiency. In addition, the Aptima SARS-CoV-2 Assay has a relatively quick turnaround time (12 hours).

However, the need for a very rapid assay that could detect SARS-CoV-2 sooner became apparent. We use the Xpert Xpress SARS-CoV-2 (Cepheid) and the Simplexa COVID-19 Direct Test (Diasorin) for this purpose.

An important aspect of accurate testing is an appropriate swabbing technique, which includes inserting a swab into the nose parallel to palate similar to the distance from the nose to the outer opening of the ear (approximately 3-4 inches), then slowly turning the swab for several seconds. ${ }^{3}$ Recent studies have shown that anterior nasal swabs have similar sensitivity and specificity as nasopharyngeal swabs. ${ }^{4}$ If a patient tests negative but has clinical symptoms consistent with COVID-19, a repeat test should be performed, as no currently available test has $100 \%$ sensitivity, viral load differs during the course of disease, and proper specimen collection influences the test result.

From the laboratory standpoint, the determination of whether the target or analyte is truly present in a specimen is key. This must first be accomplished by determining the analytical sensitivity and specificity of the tests under consideration. Early on, we compared the sensitivity and specificity of 5 tests. ${ }^{5} \mathrm{We}$ were particularly interested in false-negative reactions at that time, so the study set was enriched with CDC test-positive specimens. Since then, the FDA has released comparative data on the limit of detection, which reflects the sensitivity of commonly used assays. ${ }^{6}$ Excerpts from this listing for commonly used assays are as follows, reported in nucleic acid amplification test (NAAT) detectable units (the lower the numeric value the more sensitive the assay):

- Aptima SARS-CoV-2 assay: 600 NAAT

- cobas SARS-CoV-2: 1,800 NAAT

- Abbott RealTime SARS-CoV-2 assay: 5,400 NAAT

- Xpert Xpress SARS-CoV-2 test: 5,400 NAAT

- Simplexa COVID-19 Direct assay: 6,000 NAAT

- CDC 2019-nCoV Real-Time RT-PCR Diagnostic Panel: 18,000 NAAT

- Taqpath COVID-19 Combo Kit: 18,000 NAAT

- ID Now COVID-19: 300,000 NAAT

Our studies have demonstrated greater than 95\% sensitivity for the CDC assay, the TIBMOLBIO assay and the Xpert Xpress SARS-CoV-2 (Cepheid). The Simplexa COVID-19 Direct Test (Diasorin) and the ID Now COVID-19 (Abbott) had sensitivities below $90 \%$, which were initially deemed unacceptable for testing of inpatients at our facility. The performance of the Simplexa COVID-19 Direct Test (Diasorin) was improved if only symptomatic patients were tested with this assay, which is the current state. We considered the potential utility of the assays with less than $90 \%$ sensitivity in the ambulatory setting wherein the instructions to the patient would not change (eg, symptomatic individuals would be instructed to self-isolate and wear a mask, regardless of the test results) and possibly in settings of very low prevalence, wherein the negative predictive value exceeds 95\%, particularly if these were low-risk set- 
tings (eg, population screening). The Cleveland Clinic laboratories currently offer 5 assays, with 2 of the platforms performing rapid tests. The availability of numerous platforms allowed us to expand testing capacity. Additionally, our laboratory is presently exploring options to detect influenza $A$ and influenza $\mathrm{B}$ in conjunction with the detection of SARS-CoV-2.

We also investigated testing of saliva. Although we found testing "enhanced" saliva specimens to have a $100 \%$ positive percent agreement with testing nasopharyngeal swab specimens, the viral load in saliva was slightly lower in most patients. ${ }^{7}$ The data regarding whether the SARS-CoV-2 viral load in saliva, and hence the sensitivity of the assay, is higher or lower than in traditional nasopharyngeal specimens remains unsettled. We feel confident that in a patient with symptoms, saliva can be viewed as an acceptable alternate specimen type for the diagnosis of COVID19. However, saliva as a specimen type has not been added to our test menu, because of added complexity to the laboratory operations and since it was not found to be superior to a nasopharyngeal swab specimen in our study.

Cleveland Clinic does not offer SARS-CoV-2 antigen or antibody testing. Traditionally, antigen tests have demonstrated moderate sensitivity, requiring that negative tests in symptomatic patients be confirmed using a molecular assay. Also, numerous instances of false-positive antigen tests have been reported in the lay press, which has also diminished our interest in SARS-CoV-2 antigen detection. Similarly, the utility of SARS-CoV-2 antibody testing is limited due to cross-reactivity with other coronaviruses, false positives, false negatives, and no clear correlation with immunity.

\section{- THE IMPACT OF DATA ANALYTICS AND REPORTING ON DECISION-MAKING RELATED TO COVID-19 TESTING}

Effective data analytics were crucial to monitor test utilization, testing capacity, turnaround times, and positivity rates and to inform decisions about test allocations, priorities, and patient populations. Multiple dashboards were created that can be updated in nearreal-time and include displays of COVID-19/SARSCoV-2 test volumes filterable across the categories mentioned previously as well as turnaround times and targets for these tests. In addition, tests could be stratified by ordering location (emergency department, ambulatory, or inpatient), by hospital within the health system, and by presence or absence of symptoms. The dashboards and reports have enabled more efficient use of existing testing capacity and informed decisions regarding the extension of testing to additional patient populations or locations. Monitoring turnaround times provided important process monitoring and identified targets for troubleshooting.

\section{WHAT ARE THE IMPLICATIONS OF WHO GETS TESTED, WHERE, AND WHEN?}

To coordinate the expansion of testing groups while ensuring consideration of the logistics mentioned above, Cleveland Clinic created a COVID-19 test stewardship group with oversight by our Medical Operations group, Pathology and Laboratory Medicine Institute, and executive leadership. This group brought together the expertise of pathologists, medical operations, supply chain, information technology, nursing, primary care physicians, and continuous improvement. This group continues to meet weekly to adapt the testing strategy to new demands and to discuss data, operational constraints, implementation, and monitoring of initiatives.

Because of limited testing capacity, most health systems have resorted to prioritizing groups of patients in whom to offer testing based on priority group guidance from the CDC and Ohio Department of Health.6,7 These priority patients included those with high-risk underlying medical conditions, work-related risk, or those planned to undergo procedures and surgeries. Essential to reopening safely was developing the ability to test patients before surgeries and procedures, which resulted in a large increase in demand for testing. Operationalizing widespread COVID-19 testing on a large scale can be difficult due to a number of reasons, including managing the supply of testing kits and swabbing materials, developing capacity to collect the swab, optimizing PPE availability, and identifying an adequate number of inpatient cohorting units/beds to separate patients with COVID-19-positive, negative, and pending results. Cohorting is especially important in populations that may not be able to follow social distancing strategies, such as patients with behavioral health problems or dementia.

At Cleveland Clinic, we took a 2-pronged, iterative approach to test our emergency department and inpatient populations. For patients admitted through the emergency department, we phased in expedited (rapid) COVID-19 testing based on high-risk patient populations, such as symptomatic intensive care unit patients and labor and delivery patients, and those who needed testing prior to disposition from the emergency department, such as behavioral 
health admissions. Subsequent phases included testing patients transferring from extended care facilities, all admissions to the intensive care units and labor and delivery units (and their companions), as well as symptomatic cancer and immunosuppressed patients. As the expedited testing supply increased, testing expanded to all symptomatic admissions from the emergency department to allow for cohorting of positive patients, avoid unnecessary isolation of newly admitted patients while tests were being processed, and prevent inadvertent spread of disease. To minimize the impact on emergency department throughput and PPE use, testing was performed early in the emergency department course for patients likely to require admission. Standard PPE (goggles, masks, gloves) was maintained for all patients until COVID-19 testing resulted. Broad but targeted testing minimizes risk of transmission to caregivers and other patients, including newborns in the labor and delivery setting. ${ }^{8}$

The strategy on the inpatient side was different due to the higher capacity to perform nonrapid COVID19 testing. Expedited testing is available to symptomatic high-risk populations and specific asymptomatic inpatient populations, such as organ transplant recipients with an active organ offer, and patients requiring an emergency surgery or procedure in the next 24 hours (Figure 1). Testing before surgeries and procedures was encouraged in order to understand the risk of complications for the patient and risk of exposure to the caregivers in the operating room, and to ensure appropriate levels of PPE available in the operating room. ${ }^{9}$ Considerations when testing inpatients also include ensuring cohorting of patients under investigation, moving high-risk patients into private rooms when tests are pending results, and ensuring adequate PPE is available and being used.

Our ambulatory COVID-19 testing strategy and processes evolved quickly over a few weeks. Initially, testing was made available widely without defined criteria, but this quickly overwhelmed our operations and supplies. We quickly restricted symptomatic testing to patients with high-risk criteria. This led to a manageable volume of tests being performed, but resulted in lower-risk patients with COVID-like symptoms being presumptively diagnosed. As testing capacity increased, these restrictions were eventually lifted so that any patient with COVID-like symptoms could receive a test. Additionally, as the reopening phase led to increased demand for preoperative testing, swabbing centers were expanded to meet demand.
Patient identified as needing emergency surgery or procedure in the next 24 hours

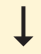

Surgeon or other provider places rapid COVID order in electronic medical record

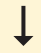

Provider selects "emergency surgery/procedure within 24 hours" under COVID order

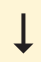

Test obtained by clinician on inpatient floor, using appropriate personal protective equipment

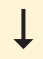

Test results followed up by surgical or procedure team

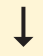

Surgery or procedure decision made by surgical or procedural team on the basis of the results

Figure 1. Rapid COVID-19 testing inpatient workflow for emergency surgeries and procedures.

\section{HOW DO OUTPATIENTS GET TESTED, AND WHAT IS THE FOLLOW-UP?}

\section{Ambulatory COVID-19 testing site considerations}

The physical location of COVID-19 testing is important because ensuring that the proper infrastructure and processes exist is imperative for both patient and caregiver safety. Considerations include:

Physical site

- Ability to test asymptomatic and symptomatic patients and caregivers

- Availability of supplies (including PPE, swabs, testing reagent, eye wash sinks, computer hardware, printers, connectivity)

- Human resources to support the processes.

The physical site should be selected based on optimal patient flow with the least amount of exposure risk. This includes identifying the ability to have a large enough space to accommodate patient and caregiver flow, segregation of testing from other clinical areas and separate spaces for donning and doffing of PPE for caregivers. Exposure risk must be mitigated for the caregivers performing the test and the other patients within the physical space, especially when testing asymptomatic and symptomatic patients in the same facility. The consideration of creating 
centralizing testing locations also requires review of PPE inventory and testing supplies across the entire organization, and ensuring there is adequate amount to support overall patient care.

A long-term testing site strategy involves identifying a space for testing at various locations that are scattered across a geographic area for patient convenience. The enterprise took advantage of lower volumes through our express and urgent care sites in the community to convert several of these partially or completely over to preprocedure testing sites for asymptomatic patients. A transition from nasopharyngeal to anterior nares swabbing and the addition of observed self-collected swabbing were 2 changes that improved throughput, conserved PPE, and optimized staffing models. A home testing kit for asymptomatic patients requiring testing prior to a surgery or procedure has been developed and awaiting FDA approval at this time. Once approved, this will greatly reduce the need for physical locations for preoperative testing.

\section{COVID-19 home monitoring program}

Patients are offered enrollment in the Cleveland Clinic COVID-19 home monitoring program following a positive test result, after hospital discharge for COVID-19, or if strongly suspected of having COVID-19 without testing by an ambulatory provider. All newly identified patients receive an outreach call with instructions on home isolation, education about COVID-19, provider screening for concerns about social support, and home safety, and an invitation to engage with the MyChart Care Companion app (Epic Systems Corp, Madison, WI). MyChart Care Companion is a patient-engagement platform available on smartphone and web-based platforms. Based on CDC guidelines, Cleveland Clinic partnered with Epic to custom-build a COVID-19 care plan in the platform to optimize engagement. Patients in the home monitoring program are monitored daily for 7 days after discharge from the hospital or for 14 days after symptom onset if they entered the program as an ambulatory patient.

Daily monitoring of patients consists of telephonic outreach from a registered nurse or allied health professional and a self-monitoring program in the MyChart Care Companion app that allows the patient to enter data about COVID-19 symptoms. Using either or both methods, patients are asked whether any of a list of symptoms are present, and whether those symptoms are getting better, getting worse, or the same. Patients reporting new or worsen- ing symptoms via the app will see a message stating that their symptoms are going to be forwarded to a clinician. After a nursing assessment, a patient may then be escalated for additional care with 2 options: (1) an urgent virtual evaluation with the patient's primary care provider or a "virtualist" physician on call, or (2) referral to the emergency department with direct handoff communication. The primary care provider or virtualist physician may order additional medications (eg, cough medications, bronchodilator), arrange for additional diagnostic testing (eg, laboratory, radiography) in a designated facility that can manage COVID-19-positive patients, or order mobile testing by a visiting paramedic or allied health professional. For patients who do not desire escalation of care and who instead choose to focus on comfort, palliative care is activated through a virtual visit assessment with urgent initiation of services if a patient reports worsening symptoms. Palliative care measures are consistent with Cleveland Clinic's ethical framework and those reported in other countries. ${ }^{10}$ Throughout the process, the patient's primary care provider or primary specialty consultant is apprised of all outreach via the electronic health record.

\section{Enabling the electronic health record to help stew- ard COVID-19 testing}

Part of the success of the testing program was due to the recognition that health information technology aspects are a critical part of the overall testing strategy. ${ }^{11}$ As the testing recommendations were and are in flux, we developed ordering processes to allow for rapid changes to meet the ever-changing workflows. A variety of techniques were utilized to provide clinical decision support at the point of ordering, including embedding the COVID-19 order in unique testing panels so that the order could be made accessible only to certain users and departments. To this end, several required and non-required questions were included in all testing orders to guide proper testing utilization and alignment with identified testing criteria, with different questions viewable for the different use cases. For example, the criteria options provided for a patient in an emergency department setting are not the same ones seen in the inpatient or ambulatory version of the order. Inpatient orders were paneled with the correct isolation precaution orders, which were preselected. Audit reports were created to track information such as ordering user, location, department, reason and test result to ensure that testing was being used as indicated. Iterations of the build also included questions to be answered by the ordering 
providers that are required by the US Department of Health and Human Services for reporting purposes.

\section{Other considerations}

As COVID-19 testing becomes more prevalent in health care settings and the community, considerations surrounding reimbursement for testing must be scrutinized. Currently, commercial payers do not generally cover testing for asymptomatic patients, and health care systems are forced to absorb these costs or are transferring the cost to patients. This is an important factor when deciding who should be tested.

A number of commercial vendors now offer COVID-19 testing for situations such as return to school, athletics, return to work, and travel, in which individuals are seeking testing when asymptomatic. These tests come at a cost for the consumer and have variable result times, which would need to be taken into account since most travel-based testing requires testing within 48 to 72 hours of departure.

Lastly, during influenza season and with patients presenting with a variety of influenza-like symptoms, it is increasingly difficult to distinguish between viral syndromes. We have elected to create a panel of testing for COVID-19, influenza, and respiratory syncytial virus once flu and respiratory syncytial virus prevalence increases in the surrounding community. Important considerations for broader testing are multiple - from empiric treatment for flu to isolation affecting bed assignment and hospital throughput to obtaining tests that can look for multiple targets such as COVID-19, flu, and respiratory syncytial virus.

\section{CONCLUSION}

Operationalizing testing for COVID-19 is a major endeavor and requires thoughtful planning. Planning must take into account a number of logistical items, including test accuracy, appropriate patient groups, using data to drive decision-making, supply chains, physical testing locations, and patient follow-up. This complex process should also include expertise from a broad range of fields such as lab medicine, operations, infectious disease, supply chain, nursing, data analysis, and information technology to ensure success.

\section{DISCLOSURES}

Dr. Simon has disclosed being on the Speakers Bureau for Alexion pharmaceuticals. All other authors report no relevant financial relationships which, in the context of their contributions, could be perceived as a potential conflict of interest.

\section{REFERENCES}

1. Hellewell J, Abbott S, Gimma A, et al. Feasibility of controlling COVID-19 outbreaks by isolation of cases and contacts. Lancet Glob Heal 2020; 8(4):e488-e496. doi:10.1016/\$2214-109X(20)30074-7

2. US Food and Drug Administration. Emergency use authorizations for medical devices. Accessed February 19, 2021. https://www. fda.gov/medical-devices/emergency-situations-medical-devices/ emergency-use-authorizations\#covid19ivd

3. Marty FM, Chen K, Verrill KA. How to obtain a nasopharyngeal swab specimen. N Engl J Med 2020; 382(22):e76. doi:10.1056/ nejmvcm2010260

4. Péré $H$, Péré $H$, Péré $H$, et al. Nasal swab sampling for SARS-CoV-2: a convenient alternative in times of nasopharyngeal swab shortage. J Clin Microbiol 2020; 58 (6):e00721-20. doi:10.1128/JCM.00721-20

5. Procop GW, Brock JE, Reineks EZ, et al. A comparison of five SARSCoV-2 molecular assays with clinical correlations. Am J Clin Pathol 2021; 155(1):69-78. doi:10.1093/ajcp/aqaa181

6. US Food and Drug Administration. SARS-CoV-2 reference panel comparative data. Accessed February 14, 2021. https://www.fda. gov/medical-devices/coronavirus-covid-19-and-medical-devices/ sars-cov-2-reference-panel-comparative-data

7. Procop GW, Shrestha NK, Vogel S, et al. A direct comparison of enhanced saliva to nasopharyngeal swab for the detection of SARSCoV-2 in symptomatic patients. J Clin Microbiol 2020; 58(11):e0194620. doi: 10.1128/JCM.01946-20. PMID:32883744

8. Sutton D, Fuchs K, D'Alton M, Goffman D. Universal screening for SARS-CoV-2 in women admitted for delivery. N Engl J Med 2020; 382(22):2163-2164. doi:10.1056/nejmc2009316

9. Lei S, Jiang F, Su W, et al. Clinical characteristics and outcomes of patients undergoing surgeries during the incubation period of COVID-19 infection. EClinicalMedicine 2020; 21:100331 doi:10.1016/j.eclinm.2020.100331

10. Roland K, Markus M. COVID-19 pandemic: palliative care for elderly and frail patients at home and in residential and nursing homes. Swiss Med Wkly 2020; 150:w20235. doi:10.4414/smw.2020.20235

11. Reeves JJ, Hollandsworth HM, Torriani FJ, et al. Rapid response to COVID-19: health informatics support for outbreak management in an academic health system. J Am Med Informatics Assoc 2020; 27(6):853-859. doi:10.1093/jamia/ocaa037

Correspondence: Anita J. Reddy, MD, MBA, Department of Critical Care Medicine, G62, Cleveland Clinic, 9500 Euclid Avenue, Cleveland, OH 44195; reddya3@ccf.org 